Wahrnehmungs- und Wertorientierte Schulentwicklung 
Christian Schroll

\section{Wahrnehmungs- und \\ Wertorientierte \\ Schulentwicklung}

Ein Evaluationskonzept zur

Qualitätsentwicklung in

allgemeinbildenden Schulen

Mit einem Geleitwort von Professor Dr. Franz Rauch

iㅡㄹ SpringerVS 
Christian Schroll

Wien, Österreich

Bei der vorliegenden Publikation handelt es sich um die veränderte Version der Dissertation zum Thema „Die Wahrnehmungs- und Wertorientierte Schulentwicklung (WWSE) als Evaluationskonzept im Rahmen der Qualitätsentwicklung in allgemeinbildenden Schulen", welche zur Erlangung des akademischen Grades Doktor der Philosophie an der Alpen Adria Universität Klagenfurt eingereicht wurde.

ISBN 978-3-658-14035-9

ISBN 978-3-658-14036-6 (eBook)

DOI 10.1007/978-3-658-14036-6

Die Deutsche Nationalbibliothek verzeichnet diese Publikation in der Deutschen Nationalbibliografie; detaillierte bibliografische Daten sind im Internet über http://dnb.d-nb.de abrufbar.

Springer VS

(C) Springer Fachmedien Wiesbaden 2016

Das Werk einschließlich aller seiner Teile ist urheberrechtlich geschützt. Jede Verwertung, die nicht ausdrücklich vom Urheberrechtsgesetz zugelassen ist, bedarf der vorherigen Zustimmung des Verlags. Das gilt insbesondere für Vervielfältigungen, Bearbeitungen, Übersetzungen, Mikroverfilmungen und die Einspeicherung und Verarbeitung in elektronischen Systemen.

Die Wiedergabe von Gebrauchsnamen, Handelsnamen, Warenbezeichnungen usw. in diesem Werk berechtigt auch ohne besondere Kennzeichnung nicht zu der Annahme, dass solche Namen im Sinne der Warenzeichen- und Markenschutz-Gesetzgebung als frei zu betrachten wären und daher von jedermann benutzt werden dürften.

Der Verlag, die Autoren und die Herausgeber gehen davon aus, dass die Angaben und Informationen in diesem Werk zum Zeitpunkt der Veröffentlichung vollständig und korrekt sind. Weder der Verlag noch die Autoren oder die Herausgeber übernehmen, ausdrücklich oder implizit, Gewähr für den Inhalt des Werkes, etwaige Fehler oder Äußerungen.

Gedruckt auf säurefreiem und chlorfrei gebleichtem Papier

Springer VS ist Teil von Springer Nature

Die eingetragene Gesellschaft ist Springer Fachmedien Wiesbaden GmbH 


\section{Vorwort des Autors}

Als Schulentwicklungsberater und Leiter des Beratungszentrums Schulentwicklung \& Leadership an der Kirchlichen Pädagogischen Hochschule Wien/Krems in Österreich bin ich häufig mit der Thematik wertorientierter und wahrnehmungsbasierter Evaluationszugänge im Zuge von Schulentwicklungsprozessen konfrontiert. Es ist mir ein großes Anliegen im Rahmen von prozessorientierter Qualitätsentwicklung, die Selbst-, Handlungs-, Sozial- und Systemkompetenz von SchulleiterInnen und LehrerInnen durch selbstgesteuerte Schulevaluationsprozesse zu unterstützen. In diesem Zusammenhang habe ich WWSE als effektives und ressourcenschonendes Evaluationstool kennengelernt und erprobt. Seit der Anwendung und Umsetzung des WWSE-Evaluationskonzeptes von Wild (2010) stellt sich für mich die Frage, inwiefern dieses Evaluationstool in allgemeinbildenden Schulen Österreichs zur nachhaltigen Qualitätsentwicklung und -sicherung beisteuern kann. Insofern liegt die Entscheidung nahe, diese Thematik einer wissenschaftlichen Auseinandersetzung zu unterziehen.

Auf dem Weg des Entstehens dieser vorliegenden Arbeit begleiteten mich Menschen, die mich mit ihren Expertisen professionell unterstützt haben. Ich möchte diesen WegbegleiterInnen meinen herzlichen Dank aussprechen: Ao. Univ.-Prof. Mag. Dr. Franz Rauch und Ass. Prof. Mag. Dr. Stefan Zehetmeier für ihre wissenschaftliche und kompetente Begleitung und Beratung, Dr. Klaus Wild für die freundschaftliche Unterstützung und Zusammenarbeit in diesem Kontext; SchulleiterInnen, LehrerInnen und WWSE-ModeratorInnen der Beispielschulen, die sich für Interviews zur Verfügung gestellt haben und allen FreundInnen, die mir beim Entstehungsprozess dieses Buches unterstützend beigestanden sind.

Mag. Dr. Christian Schroll 


\section{Geleitwort}

Aktuelle Entwicklungen der Rahmenvorgaben in der österreichischen Schullandschaft fokussieren auf Kompetenzentwicklung und Bildungsstandards. Damit verbunden werden Schulen dazu verpflichtet, Maßnahmen des Qualitätsmanagements zu konzipieren und durchzuführen. Dies schließt Schulentwicklung und Evaluation entscheidend mit ein.

Im vorliegenden Buch stehe die Fragen im Zentrum, inwieweit das Konzept der Wahrnehmungs- und Wertorientierten Schulentwicklung (WWSE) nach Wild (2010) ${ }^{1}$ als Evaluationsinstrument für innere Schulentwicklung genützt werden kann, was es leistet und welche Verbesserungspotentiale bestehen. Diesen Fragen wird in drei Fallstudien, basierend auf Einzel- und Gruppeninterviews und einer Cross-Case-Analyse, nachgegangen. Die überzeugend herausgearbeiteten Hypothesen geben deutliche Hinweise auf die Relevanz des Ansatzes, beschreiben aber auch die Bedeutung förderlicher und hemmender Bedingungen sowie Verbesserungspotentiale von WWSE. Der Text mündet in praxisbezogene Empfehlungen, bezogen auf die Aus-, Fort- und Weiterbildung von WWSEModeratorInnen zu SchulentwicklungsberaterInnen, auf die Beratung und Begleitung von Führungskräften im WWSE-Prozess sowie auf die Neugestaltung der WWSE-Fragebögen.

Leserinnen und Lesern wird über die eigenständige empirische Forschungsarbeit hinausgehend ein gelungener Überblick über aktuelle Forschungsergebnisse zu Schulqualität und Schulentwicklung im deutschen Sprachraum geboten. Das Evaluationsmodell WWSE selbst wird in bestehende Evaluationskonzeptionen und Vorgaben eingebettet und theoretisch verortet.

\footnotetext{
${ }^{1}$ Wild, K. (2010). Wahrnehmungsorientierte Schulentwicklung. Innere Schulentwicklung unter Berücksichtigung der Wahrnehmung von Schulqualität durch Lehrkräfte. Winzer: Verlag Josef Duschl. 2. Auflage.
} 
Das Buch kann daher vielfältige Anregungen für Praxis und Evaluation bzw. Forschung sowohl für Lehrkräfte und Schulleitungen als auch für SchulentwicklungsberaterInnen und MitarbeiterInnen in der LehrerInnenfort- und weiterbildung bieten.

Prof. Dr. Franz Rauch, Alpen-Adria-Universität Klagenfurt 


\section{Inhaltsverzeichnis}

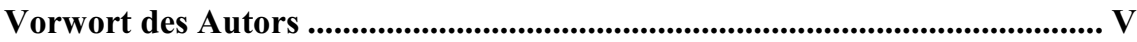

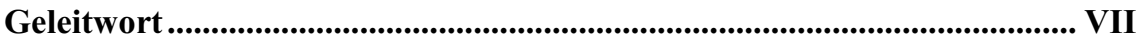

Inhaltsverzeichnis ..................................................................................................

Abbildungsverzeichnis.......................................................................................

Abkürzungsverzeichnis .......................................................................................

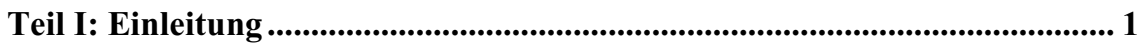

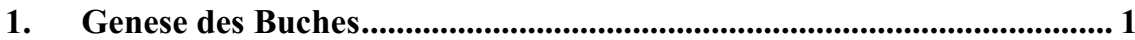

2. Ausgangspunkt und Entwicklung der Fragestellung ................................. 3

Teil II: Theoretische Grundlagen...................................................................... 7

1. Qualität im Bildungswesen ................................................................... 7

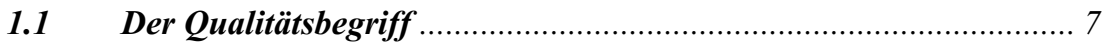

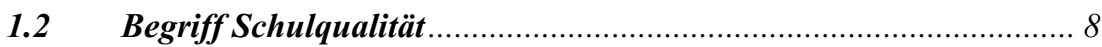

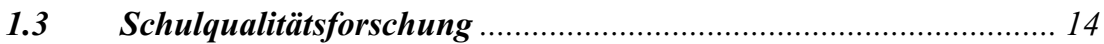

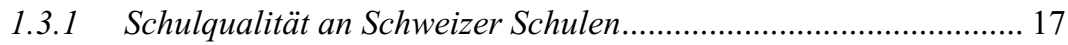

1.3.2 Schulwirksamkeitsstudie nach Purkey und Smith ........................ 19

1.4 Qualität und Relevanz von Standards im Bildungskontext ........... 21

1.4.1 Internationale Zugänge ........................................................... 22

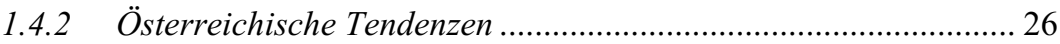

$1.5 \quad$ Schulqualität Allgemeinbildung (SQA) …….............................. 30

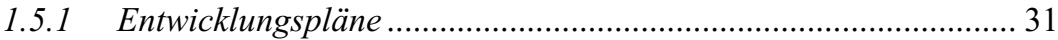

1.5.2 Bilanz- und Zielvereinbarungsgespräche .................................. 32

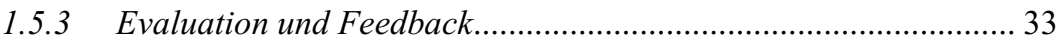

1.5.4 Entwicklungsberatung in Schulen ................................................ 34 
1.6 Qualitätsinitiative Berufsbildung (QIBB) ..................................... 36

1.7 Kurzzusammenfassung und Folgerungen...................................... 37

2. Schwerpunkte innerer Schulentwicklungsforschung.............................. 41

$2.1 \quad$ Organisationsentwicklung ....................................................... 41

2.2 Selektierte Forschungsergebnisse Organisationsentwicklung ....... 44

2.2.1 Organisationsentwicklung in Theorie und Praxis........................ 48

2.2.2 Organisationsentwicklung in lernenden Organisationen .............. 51

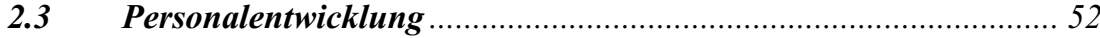

$2.4 \quad$ Selektierte Forschungsergebnisse Personalentwicklung ............... 53

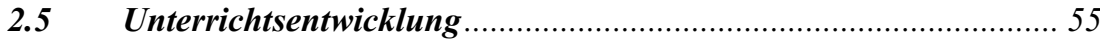

2.6 Selektierte Forschungsergebnisse Unterrichtsentwicklung ........... 57

2.7 Schulentwicklungsforschungsansatz in Österreich ....................... 59

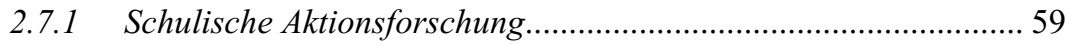

2.7.2 Entwicklung und Stand schulischer Aktionsforschung................. 61

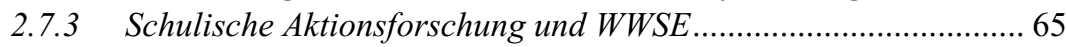

2.7.4 Zusammenfassung und Resümee ............................................... 67

3. Zur Bedeutung von Evaluation im Bildungswesen ................................ 71

3.1 Begriffserklärung Evaluation …….................................................. 71

3.2 Formen der (Schulqualitäts-)Evaluation ...................................... 74

3.2.1 Formative und summative Evaluation ........................................ 75

3.2.2 Interne Schulevaluation - Selbstevaluation ................................. 75

3.2.3 Externe Schulevaluation - Fremdevaluation …............................ 77

3.2.4 Ergebnis- und Prozessevaluation .................................................. 79

3.2.5 Zusammenfassende Perspektiven ............................................... 80

3.3 Evaluationsmethoden und Evaluationstechniken ........................ 83

3.4 Ausgewählte Konzepte der Selbstevaluation ................................. 86

3.4.1 Selbstevaluation in Schulen ........................................................... 86

3.4.2 Qualität durch Evaluation und Entwicklung.............................. 87

3.5 Ausgewählte Formen der Fremdevaluation .................................. 88 


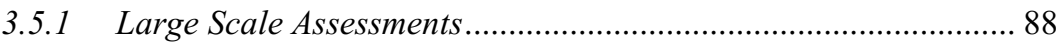

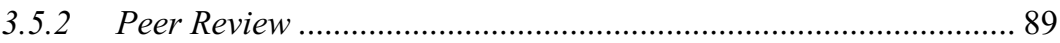

3.5.3 Lernstandserhebungen im Rahmen von Bildungsstandards ......... 90

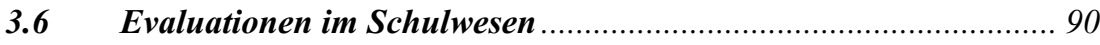

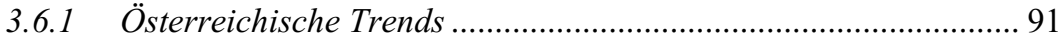

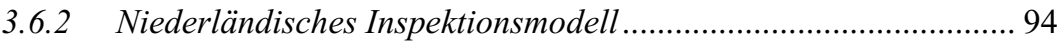

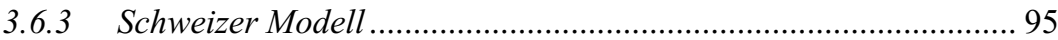

3.6.4 Evaluationen in Schulen Bayerns .............................................. 97

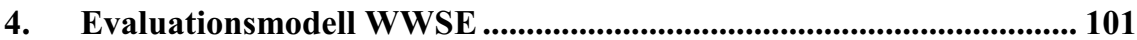

4.1 Theoretische Fundierung und Zielsetzung ................................ 102

4.2 Durchführung von WWSE an einer Beispielschule ................... 105

4.3 Datenauswertung einer Beispielschule ...................................... 107

4.4 Relevanz der Daten einer Beispielschule ..................................... 113

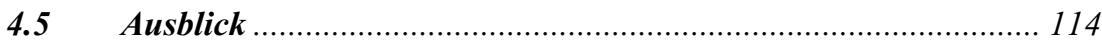

5. Werteorientierung in der Schulentwicklung .......................................... 117

5.1 Prozess- und Organisationsethik in der Schule ......................... 117

5.2 Prozessethische Herausforderungen ............................................ 119

5.2.1 Prozessethik organisiert (kollektive) Selbstreflexion .................. 120

5.2.2 Selbstreflexion betrifft Individuen und Kollektive ....................... 121

5.2.3 Selbstreflexion benötigt ein Sich-in-Distanz-Setzen..................... 121

5.2.4 Kollektive ethische Entscheidungen - Überforderungsentlastung122

5.2.5 Einrichtung von kollektiven ethischen Entscheidungsprozessen. 122

5.2.6 Repräsentationsverfahren - Ermöglichung Feedbackschleifen .. 123

5.3 Prozessethische Entscheidungsverfahren organisieren ............... 123

5.4 Organisationsethische Überlegungen............................................ 124

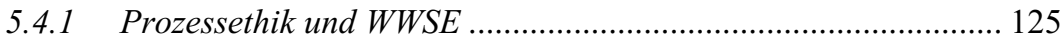

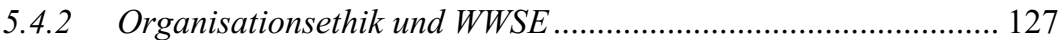

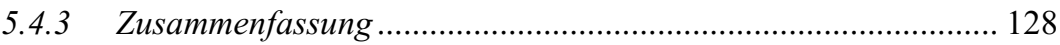

5.5 Organisationskultur in der Schule ................................................ 129 


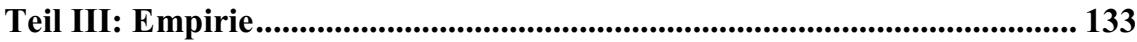

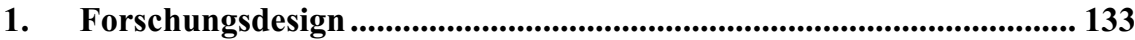

$1.1 \quad$ Dimensionsbestimmungen ....................................................... 133

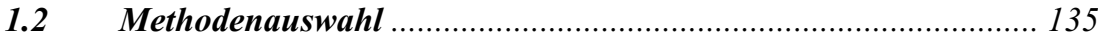

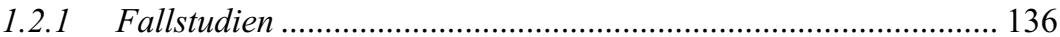

1.2.2 Charakteristik der Fallstudien ............................................... 138

$1.3 \quad$ Falldefinition und -bearbeitung ................................................ 140

$1.4 \quad$ Konstruktion der Erhebungsinstrumente …............................... 142

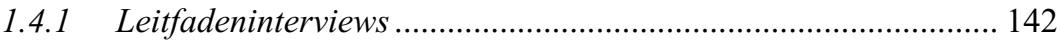

1.4.2 Gruppeninterviews .............................................................. 143

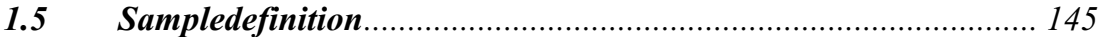

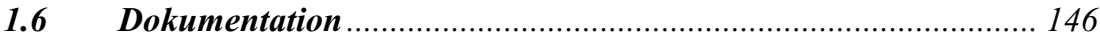

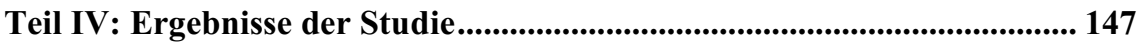

1. Darstellung der Fallstudien ..................................................................... 147

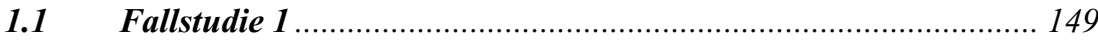

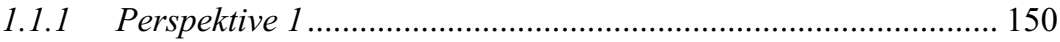

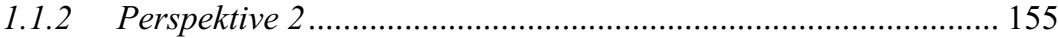

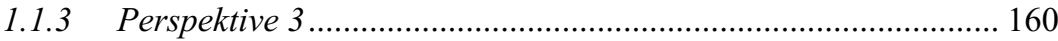

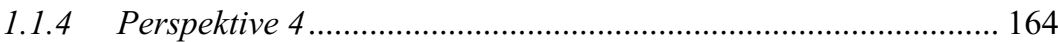

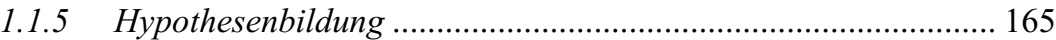

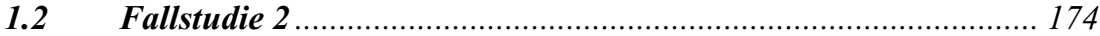

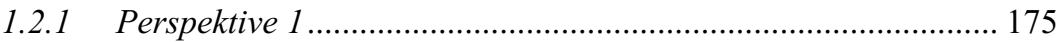

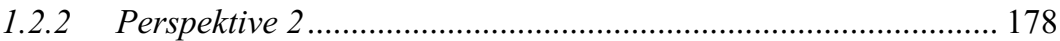

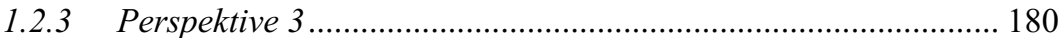

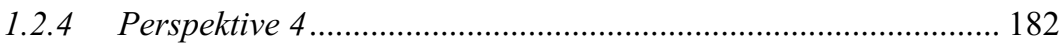

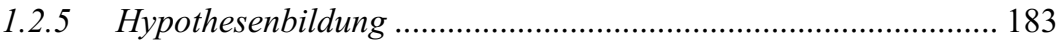

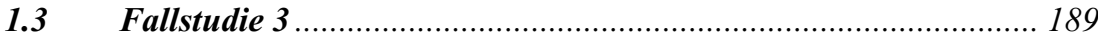

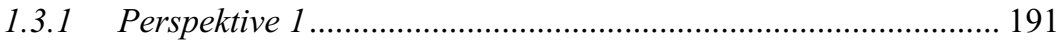

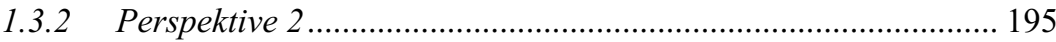

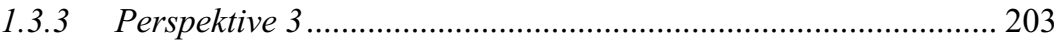




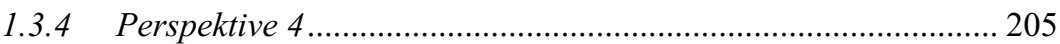

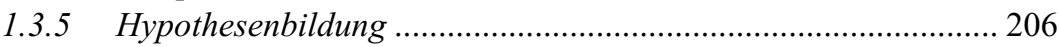

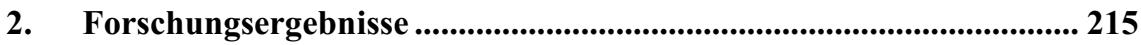

2.1 Cross-Case-Analyse und Hypothesenvergleich …....................... 215

2.1.1 Bedeutung von schulischer Qualitätsentwicklung...................... 218

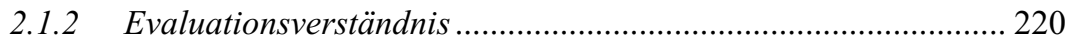

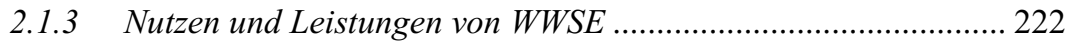

2.1.4 Professionalisierung der WWSE-ModeratorInnen ..................... 224

2.1.5 Rolle und Funktion der Schulleitung im WWSE-Prozess ............ 226

2.1.6 Gestaltung und Adaptierung der WWSE-Fragebögen ................ 228

2.1.7 Soziale Aspekte ...................................................................... 228

2.1.8 Werte und ethische Prinzipien ................................................... 229

2.1.9 Beratung und Begleitung von Schulen ...................................... 230

2.1.10 WWSE als Teil von SQA ................................................... 231

$2.2 \quad$ Resümee und Zusammenfassung Cross-Case-Analyse................ 231

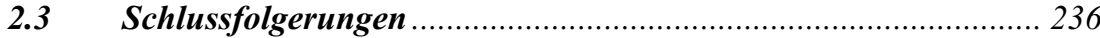

2.3.1 Relevanz, Leistung und Nutzen von WWSE............................... 236

2.3.2 Förderliche und hemmende Bedingungen .................................. 241

2.3.3 WWSE-Verbesserungs- und Entwicklungspotenziale.................. 243

3. Praxisbezogener Ausblick und Empfehlungen ........................................ 247

3.1 Aus-, Fort- und Weiterbildung von WWSE-ModeratorInnen ...... 247

3.2 Beratung und Begleitung von Führungskräften bei WWSE ........ 251

3.3 Neugestaltung der WWSE-Fragebögen ................................... 252

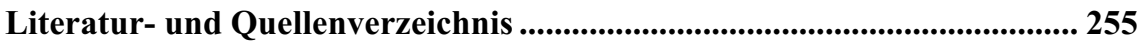




\section{Abbildungsverzeichnis}

Abbildung 1: Qualitätssicherungsprozess in Schulen nach bm:bwk 2004, S. 5

Abbildung 2: Definitionsansätze Qualität nach Garvin (1984) in Benes/Groh

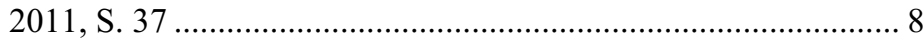

Abbildung 3: Kriterienkatalog nach Purkey und Smith (1991)....................... 19

Abbildung 4: Qualitätsregelkreis QIBB (ebd.)............................................. 37

Abbildung 5: Verfahren der Organisationsentwicklung nach Holtappels

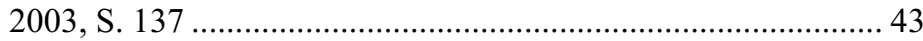

Abbildung 6: Übersicht über Modelle der Feldarbeit nach Grossmann et al. 2007, S. 81f

Abbildung 7: Entscheidungsfelder in der Evaluation nach Klieme 2005,

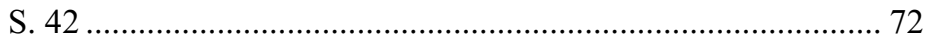

Abbildung 8: Evaluationszwecke von Altrichter 2010, S. 174 ....................... 79

Abbildung 9: Ziele interner und externer Evaluation nach Holtappels 2003,

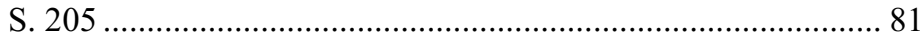

Abbildung 10: Evaluationsmatrix nach Klopsch 2009, S. 44 ......................... 82 Abbildung 11: Unterschiedliche Evaluationsziele nach Sprick 2008, S. 65 ..... 82 Abbildung 12: Prozess- und Ergebnisevaluation nach Fend 2008, S. 123 ......... 83 Abbildung 13: Aufbau/Struktur von Selbstevaluation in der Praxis

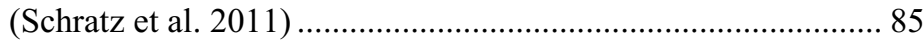

Abbildung 14: ABC-Modell der 3 Evaluationsphasen nach Weerts (2007) ...... 95 Abbildung 15: Qualitätsbereiche zur externen Evaluation nach Schieß1

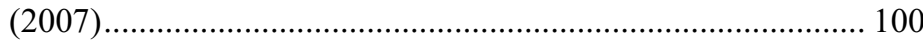

Abbildung 16: Auswertungsbogen von Wild (2010) .................................... 104

Abbildung 17: WWSE-Ergebnis Beispielschule Organisationsentwicklung ... 109 Abbildung 18: WWSE-Ergebnis Beispielschule Personalentwicklung A ....... 110 Abbildung 19: WWSE-Ergebnis Beispielschule Personalentwicklung B ........ 111 Abbildung 20: WWSE-Ergebnis Beispielschule Unterrichtsentwicklung ....... 112 Abbildung 21: Schwerpunktsetzung der Beispielschule ............................... 113 Abbildung 22: Prozessethische Verfahren nach Krainer/Heintel 2010, S. $207 \mathrm{ff}$.

Abbildung 23: Prozessethik (Krainer/Heintel 2010) vs. WWSE (Wild 2010). 127 Abbildung 24: Levels of Culture (Schönig 2009, S. 153) .............................. 131 Abbildung 25: Darstellung der Fälle im Überblick........................................... 141 
Abbildung 26: Ablaufmodell der Gruppendiskussion nach Mayring 2002, S. 79 144

Abbildung 27: Perspektiven zur Fallanalyse 1 150

Abbildung 28: Perspektiven zur Fallstudie 2 175

Abbildung 29: Perspektiven zur Fallstudie 3 190

Abbildung 30: Vergleichsdarstellung: Kategorien der drei Einzelfälle Typologien 218

Abbildung 31: Inhalte Begleitung und Beratung von SchulleiterInnen im WWSE-Prozess. 252 


\section{Abkürzungsverzeichnis}
APS
Allgemeinbildende Pflichtschule
BIFIE
Bundesinstitut Bildungsforschung, Innovation und Ent- wicklung des österreichischen Schulwesens
BINE
Bildung für nachhaltige Entwicklung - Innovation - in der LehrerInnenbildung
BMBF
Bundesministerium für Bildung und Frauen
bm:bwk
Bundesministerium für Bildung, Wissenschaft und Kultur
bm:ukk
Bundesministerium für Unterricht und Kunst
bzw. beziehungsweise
COOL
Cooperatives Offenes Lernen in Berufsbildenden Höhe- ren Schulen
d.h.
das heißt
ebd.
eben da
EBIS
Entwicklungsberatung in Schulen
EFQM
European Foundation for Quality Management
ENSI
Environment and School Initiatives
EOS
Entwicklung - Organisation - System
fBM
Fachbezogenes Bildungsmanagement
IMST
Innovationen machen Schulen Top
INIS
Internationales Netzwerk innovativer Schulsysteme
ISE
Institut für Schulentwicklung und Evaluation Nürnberg
ISP
Institutionelles Schulentwicklungsprogramm
MMS
Modulansatz zur Selbstevaluation von Schulentwicklung
o.ä.
oder ähnliche(s)
$\mathrm{OE}$
Organisationsentwicklung
PE
Personalentwicklung
PFL
Pädagogik und Fachdidaktik für LehrerInnen
$\mathrm{PH}$
Pädagogische Hochschule(n)
ProFil
Professionalität im Lehrberuf 


$\begin{array}{ll}\text { PSE } & \text { Pädagogische Schulentwicklung } \\ \text { Q2E } & \text { Qualität durch Evaluation und Entwicklung } \\ \text { QIBB } & \text { Qualitätsinitiative Berufsbildung } \\ \text { Q.I.S } & \text { Qualität in Schulen } \\ \text { RST } & \text { Regulier School Toezicht } \\ \text { SCOL } & \text { Social Competence and Organizational Learning } \\ \text { SEIS } & \text { Selbstevaluation in Schulen } \\ \text { sog. } & \text { sogenannt(e) } \\ \text { SQA } & \text { Schulqualität Allgemeinbildung } \\ \text { TQM } & \text { Total Quality Management } \\ \text { u.a. } & \text { unter anderem } \\ \text { UE } & \text { Unterrichtsentwicklung } \\ \text { usw. } & \text { und so weiter } \\ \text { u.v.m } & \text { und vieles mehr } \\ \text { vs. } & \text { versus } \\ \text { WWSE } & \text { Wahrnehmungs- und Wertorientierte Schulentwicklung } \\ \text { z.B. } & \text { zum Beispiel }\end{array}$

